\title{
POLYARTHRITIS ASSOCIATED WITH ACUTE HISTOPLASMOSIS
}

\author{
Vivian de Aquino Medici ${ }^{1}$, Marinara Lopes Chaves $^{1}$, Luana Oliveira da Silva ${ }^{1}$, Flavio Barboza ${ }^{1, \star}$ \\ 1.Universidade Federal de Mato Grosso, Sinop (MT), Brazil. \\ *Corresponding author: flavio.fbarboza@uol.com.br
}

\section{BACKGROUND}

Histoplasmosis is a systemic fungal infection, with characteristics of a granulomatous disease and predilection for the lung, caused by the saprophytic fungus Histoplasma capsulatum. It is found in birds and bats, in caves and rural environments. Contagion by this fungus occurs through the inhalation of spores, therefore, the lung is the most affected organ. In some patients (mainly elderly and immunosuppressed) the disease can manifest in a disseminated form, with fever, weight loss, anemia, skin and joint lesions. The classic joint picture is septic arthritis, but it can present as oligoarthritis of the lower limbs (mimicking acute sarcoidosis_-Lofgran's syndrome) or rarely as polyarthritis reactive to systemic infection.

\section{CASE REPORT}

Female, 30 years old, under investigation by a pulmonologist due to acute chest discomfort, fever and joint pain, with no other apparent complaints. Chest X-ray showed nodule in the left lower lobe. The tomography showed a $1.2 \times 1.5 \times 1.43 \mathrm{~cm}$ spiculated nodule (Figure 1), with apical and bilateral thickening of the pleura and mediastinal lymph node enlargement. A lung biopsy was performed for histopathological analysis of the nodule, which showed caseous necrosis. Biopsy culture tests were performed, with negative results for histoplasmosis, cryptococcosis and paracoccidioidomycosis. Infectious screens were excluded for HIV, hepatitis, negative PPD, and negative sputum culture. After three months of severe polyarticular pain, the patient was referred for evaluation of rheumatologic disease to exclude the diagnosis before starting the RIPE regimen. Physical examination showed polyarthritis of large and small joints, worse in ankles and wrists. Laboratory tests showed elevated CRP and ESR, negative ANA and ANCA, negative rheumatoid factor and anti-CCP, platelets, mild anemia, negative blood cultures and negative serology for cryptococcosis and paracoccidioidomycosis, but with anti-histoplasma band $\mathrm{M}+$ antibodies. Once the diagnosis of histoplasmosis was assumed, Itraconazole was started, associated with low doses of corticosteroids to improve the severe polyarthritis, leading to clinical improvement in the patient.

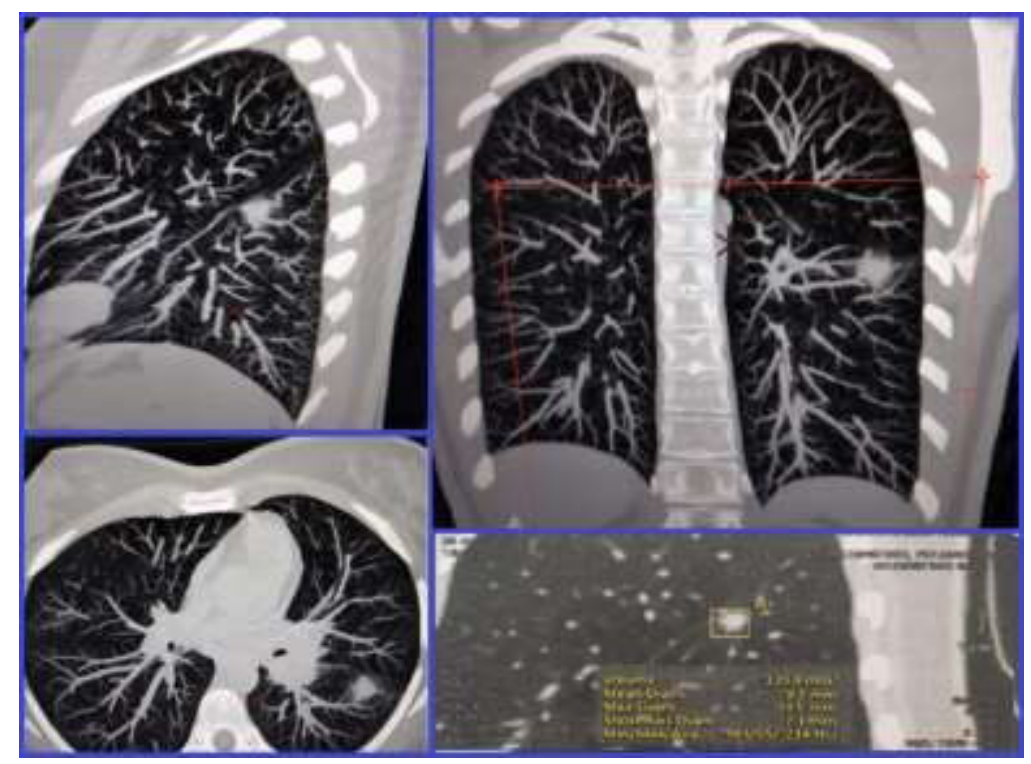

Figure 1. Spiculated nodule in left lung. 


\section{CONCLUSION}

In the investigation of polyarthritis, infectious etiologies should not be forgotten, even without the usual presentation (septic arthritis), especially when there is evidence of caseous necrosis in the histopathology. The investigation must be complete for fungi, even with negative cultures. In the end, the use of low-dose corticosteroids may be necessary as an adjunct to antifungal treatment for the initial improvement of joint symptoms. Some patients may need a longer use of steroids due to relapse of the arthritis, which may be associated with hydroxychloroquine during treatment.

\section{KEYWORDS}

Fungal infection, Polyarthritis, Histoplasmosis. 